International Journal of Computer Networks \& Communications (IJCNC) Vol.3, No.6, November 2011

\title{
Performance Evaluation of THe LAR-1P ROUTE DisCovery AlgorithM
}

\author{
Hussein Al-Bahadili and Ali Maqousi \\ Faculty of Information Technology, Petra University \\ P. O. Box 961343, Amman 11196, Jordan \\ (hbahadili@uop.edu.jo, amaqousi@uop.edu.jo)
}

\begin{abstract}
The location-aided routing scheme 1 (LAR-1) and probabilistic algorithms were combined together into a new algorithm for route discovery in mobile ad hoc networks (MANETs) called (LAR-1P) [1]. Simulation results demonstrated that, on network scale, for a uniform random node distribution and for a specific simulation setup (time); the LAR-1P algorithm reduces the number of retransmissions as compared to LAR-1 at a cost of insignificant reduction in the average network reachability. However, on zone scale, the algorithm provides an excellent performance in high-density zones, while in low-density zones; it almost preserves the performance of LAR-1. This paper provides a detail analysis of the performance of the LAR-1P algorithm through various simulations, where the actual numerical values for the number of retransmissions and reachability in high- and low-density zones are estimated to demonstrate the effectiveness and significance of the algorithm and how it provides better performance than LAR-1 in high-density zones. Furthermore, the effect of the total number of nodes on the average network performance is also investigated.
\end{abstract}

\section{KEYWORDS}

LAR-1P, LAR-1, probabilistic algorithm, broadcast MANET, pure flooding, flooding optimization algorithms, routing protocols, route discovery algorithms.

\section{INTRODUCTION}

In a mobile ad hoc network (MANET), data packets are forwarded to other mobile nodes on the network through reliable and efficient dynamic routing protocols [1], which are part of the network layer software that is responsible for deciding which output route a packet should be transmitted on. Dynamic routing protocols (e.g., the dynamic source routing (DSR) [2], ad hoc on-demand distance vector (AODV) [3], zone routing protocol (ZRP) [4]) consist of two main phases; these are: route discovery and route maintenance. Route discovery is used when a source node desires to send a packet to some destination node and does not already have a valid route to that destination; in which the source initiates a route discovery process to locate the destination. It broadcasts a route request (RREQ) packet to its neighbours, which then forward the request to their neighbours, and so on until the expiration of the packet. During the forwarding process, the intermediate nodes record in their route tables the address of the node from which the first copy of the broadcast packet is received. Once the RREQ reaches the destination, the destination responds with a route reply (RREP) packet back to the source through the route from which it first received the RREQ. Otherwise, if the RREQ packet expired before reaching its destination, then the node at which it expires, sends a route error (RERR) packet back to the source to initiate a new route discovery process [3].

Pure flooding is the earliest, simplest, and reliable mechanism proposed in the literature for route discovery in MANETs [5, 6]. In pure flooding, each node rebroadcasts the message to its neighbours upon receiving it for the first time, starting at the source node. Although it is simple

DOI : 10.5121/ijcnc.2011.3604 
and reliable, pure flooding is costly where it costs $N$ transmissions in a network of $N$ reachable nodes. In addition, pure flooding in wireless networks, using the IEEE 802.11 protocol, results in serious redundancy, contention, and collisions in the network; such a scenario has often been referred to as the broadcast storm problem (BSP) [7].

A variety of flooding optimization algorithms have been developed to alleviate the effects of BSP during route discovery in MANETs by reducing the number of redundant retransmissions without significantly affecting network reachability. Examples of such algorithms include the LAR schemes 1 (LAR-1) [8,9] and probabilistic broadcast [10, 11, 12], which we concern with in this work.

The performance of the LAR-1 scheme significantly suffers from the high number of redundant retransmissions in high-density zones (networks) [8]. While the probabilistic algorithm usually provides excellent performance in high-density zones, by significantly reducing the number of retransmissions with almost no effect on network reachability, and that of course subject to proper retransmission probability $\left(p_{t}\right)$ adjustment mechanism $[5,6,11]$. Recently, a new route discovery algorithm was proposed that combines the LAR-1 and probabilistic algorithms, and it was called LAR-1P [1]. In this algorithm, when receiving a request message, an intermediate node within the request zone rebroadcasts the message with dynamically adjusted $p_{t}$. LAR-1P combines the better of the two algorithms, in low-density zones; the nodes assigned a $p_{t}$ of unity or close to unity, so that the algorithm acts as LAR-1, while a satisfactory $p_{t}$ can be assigned in high-density zones, which means the algorithm acts as probabilistic.

The simulation results in [1] demonstrated that for a uniform random node distribution on specific network area and for a certain simulation setup, LAR-1P requires less number of retransmissions in comparison with LAR-1 inflicting insignificant reduction in average network reachability. The reduction in reachability can be avoided by proper $p_{t}$ adjustment at lowdensity zones. Furthermore, LAR-1P can provide better average performance if the route discovery processes is performed more in high-density zones than in low-density zones.

This paper analyzes in details the performance of the LAR-1P algorithm using the MANET simulator (MANSim) [13]. First, the effect of node densities (total number of nodes on the network divided by the network area) on the performance of LAR-1P is investigated and compared against other algorithms like pure flooding, dynamic probabilistic, and LAR-1. Second, the effects of zones densities, for a certain node density $\left(4 \times 10^{-4}\right.$ node $\left./ \mathrm{m}^{2}\right)$ and one particular source node (node 1), on performance are investigated for both LAR-1 and LAR-1P algorithms.

The rest of the paper is organized as follows. Literature review is presented in Section 2. Pure, probabilistic, and LAR-1 algorithms and computation procedures implemented in MANSim are described in Section 3. Simulation results and performance analysis and discussion are presented in Section 4. Finally, Section 5 summarizes the conclusions and recommendations for future work.

\section{Literature REVIEW}

In this section, we present some of the work that is related to the LAR-1P, LAR-1, and probabilistic algorithms to provide the reader with development steps in those algorithms. LAR$1 \mathrm{P}$ was first proposed by Al-Bahadili et al [14]. It was initially proposed with fixed $p_{t}$. The detail description of the fixed probability LAR-1P with some analytical analysis can be found in $[14,15]$. Later on, Al-Bahadili [1] developed and compared the performance of a dynamic probability LAR-1P, which will be described in the next section. This will be the third published paper discussing this promising route discovery algorithm. 
Location information was first used by Ko and Vaidya [8] to develop two different LAR schemes, namely, LAR-1 and LAR-2 to reduce flooding overhead in ad hoc networks. Li et al [16] presented a modified version of the LAR protocol, which was called LAKER (location aided knowledge extraction routing) protocol for MANETs. Geography based ad hoc on demand disjoint multipath (GAODM) routing protocol was proposed by Zeng et al [17] to be used instead of pure flooding in ad hoc networks to enhance their performance. Bolena and Camp [9] and Vyas and Mahgoub [18] combined location information and mobility feedback and pattern to create new routing protocol to reduce routing overheads. The results showed reduction in the routing messages when the information of predictable mobility feedback and pattern of the mobile nodes are utilized.

For the probabilistic algorithm, it was first used by Haas et al [14] for route discovery in ad hoc networks, and they called it a gossip-based route discovery (GOSSIP1) approach. They used a predefined $p_{t}$ to decide whether or not a node forwards the RREQ packets. Later on, Haas et al developed a modified protocol, in which they gossip with $p_{t}=1$ for the first $h$ hops before continuing to gossip with $p_{t}<1$. Their results showed that they can save up to $35 \%$ message overhead compared to simple flooding.

S. Tseng et al [7] investigated the performance of the probabilistic flooding for various network densities. They presented results for three network parameters, namely, reachability, saved rebroadcast, and average latency, as a function of $p_{t}$ and network density. Sasson et al [19] developed probabilistic algorithm in which nodes would dynamically adjust their $p_{t}$ based on local topology information. Kim et al [20] introduced a probabilistic broadcasting scheme in which a node dynamically adjusts its $p_{t}$ according to its additional coverage area. The additional coverage is estimated by the distance from the sender. The simulation results showed these schemes generate fewer rebroadcasts than pure flooding approach. It also incurs lower broadcast collision without sacrificing high reachability.

Scott and Yasinsac [21] presented a dynamic probabilistic solution that is appropriate to solving BSPs in dense mobile networks. The approach can prevent broadcast storms during flooding in dense networks and can enhance comprehensive delivery in sparse networks. Barret et al [22] introduced a probabilistic routing protocol for sensor networks, in which a sensor decides to forward a message with $p_{t}$ that depends on various parameters, such as the distance of the sensor to the destination, the distance of the source sensor to the destination, or the number of hops a packet has already traveled. Their results concluded that protocols using network information perform better than protocols that do not, even in the presence of strong noise.

Viswanath and Obraczka [23] developed an analytical model to study the performance of plain and probabilistic flooding in terms of its reliability and reachability in delivering packets showing that probabilistic flooding can provide similar reliability and reachability guarantees as plain flooding at a lower overhead. A probabilistic scheme that dynamically adjusts $p_{t}$ as per node distribution and node movement was proposed by Zhang and Agrawal in [24]. The scheme combines between probabilistic and counter-based approaches. Their simulation results showed that the new scheme performs better than the two algorithms.

Abdulai et al [25] studies the performance of the AODV protocol over a range of possible $p_{t}$. They focused on the route discovery part of the routing algorithm, they modified the AODV implementation to incorporate $p_{t}$; the RREQ packets are forwarded in accordance with a predetermined $p_{t}$. Simulation results showed that setting efficient $p_{t}$ has a significant effect on the general performance of the protocol. The results also revealed that the optimal $p_{t}$ for efficient performance is affected by the prevailing network conditions such as traffic load, node density, and node mobility. Abdulai et al [33] also proposed two probabilistic methods for ondemand route discovery, that is simple to implement and can significantly reduce the overhead involved in the dissemination of RREQs. The two probabilistic methods are: the adjusted 
probabilistic (AP) and the enhanced adjusted probabilistic (EAP) which address the broadcast storm problem in the existing OADV routing protocols.

Bani-Yassein et al [5] proposed a dynamic probabilistic algorithm to improve network reachability and saved rebroadcast. The algorithm determines $p_{t}$ by considering the network density and node movement. This is done based on locally available information and without requiring any assistance of distance measurements or exact location determination devices. Bani Yassein et al, in [6] combined probabilistic and knowledge based approaches on the AODV protocol to enhance the performance of existing protocol by reducing the communication overhead incurred during the route discovery process. The simulation results revealed that equipping AODV with fixed and adjusted probabilistic flooding helps to reduce the overhead of the route discovery process whilst maintaining comparable performance levels in terms of saved rebroadcasts and reachability as achieved by conventional AODV. Moreover, the results indicated that the adjusted technique results in better performance compared to the fixed one.

Khan et al [27] proposed a coverage-based dynamically adjusted probabilistic scheme and compared its performance with simple and fixed probabilistic schemes. The proposed scheme keeps up the reachability of simple flooding while maintaining the simplicity of probability based schemes. Hanash et al [28] proposed a dynamic probabilistic approach that can efficiently reduce broadcast redundancy in MANETs. The algorithm dynamically calculates $p_{t}$ according to $k$. The simulation results showed that broadcast redundancy can be significantly reduced through their approach while keeping the reachability high.

Al-Bahadili [11] developed a new $p_{t}$ adjusting model, in which the neighborhood densities are divided into three regions (low, medium, and high). The performance of the new model was evaluated and compared against pure and other probabilistic algorithms. The model enhances the performance of probabilistic broadcast by reducing the number of transmissions while keeping almost the same network reachability.

\section{DESCRIPTION OF THE LAR-1P ALgORITHM}

This section presents the detail description of the LAR-1P algorithm, but before that we provide a brief description to the LAR-1 and probabilistic algorithms.

\subsection{The Location-Aided Routing Scheme 1 (LAR-1) Algorithm}

The LAR-1 algorithm is based on two important concepts, namely, the expected zone (EZ) and the request zone (RZ). For EZ, consider a source node $S$ that needs to find a route to a destination node $\mathrm{D}$. Assume that $\mathrm{S}$ knows that $\mathrm{D}$ was at location $\mathrm{L}$ at time $t_{0}$, and the current time is $t_{l}$. Then, EZ of $\mathrm{D}$, from the viewpoint of $\mathrm{S}$ at time $t_{l}$, is the region that expected by $\mathrm{S}$ to contain D at time $t_{1}$. S can determine EZ based on the knowledge that D was at location $\mathrm{L}$ at time $t_{0}$, and travels with speed $u$, then $\mathrm{S}$ may assume that EZ is the circular region of radius $u\left(t_{1}\right.$ $\left.-t_{0}\right)$, as illustrated in Figure 1a.

If the actual speed happens to be larger than the average, then the destination may actually be outside EZ at time $t_{l}$ and vice versa. Thus, EZ is only an estimate made by $\mathrm{S}$ to determine a region that potentially contains $\mathrm{D}$ at time $t_{l}$. In general, it is possible to define $u$ to be the average speed $\left(u_{\text {avg }}\right)$, the maximum speed $\left(u_{\max }\right)$, or some other measure of the speed distribution $\left(u_{d i s}\right)$. If $\mathrm{S}$ does not know a previous location of $\mathrm{D}$, then $\mathrm{S}$ cannot reasonably determine EZ, in this case, the entire region that may potentially be occupied by the ad hoc network is assumed to be EZ, and the algorithm reduces to the basic flooding algorithm. In general, having more information regarding mobility of D can result in a smaller EZ. For instance, if S knows that D is moving north, then the circular EZ in Figure 1a can be reduced to a semi-circle, as in Figure $1 b$ [8]. 


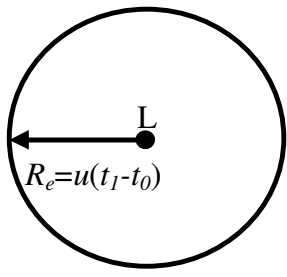

(a)

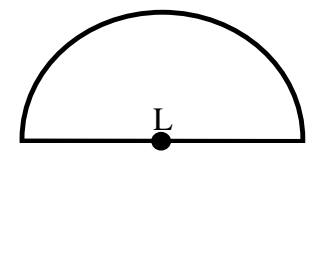

(b)

Figure 1. Examples of expected zones.

For RZ, again, consider $\mathrm{S}$ needs to determine a route to D. The LAR-1 algorithm uses pure flooding with one modification. S defines (implicitly or explicitly) a RZ for the RREQ that contains both $\mathrm{S}$ and $\mathrm{D}$ and the locations of $\mathrm{S}$ and $\mathrm{D}$ as its opposite corners. A node forwards a RREQ only if it belongs to RZ (unlike pure flooding). To increase the probability that the RREQ will reach D, RZ should include EZ (Figure 2a). Additionally, RZ may also include other regions around RZ [8]. Note that the probabilities of finding a path (in the first attempt) can be increased by increasing the size of RZ. However, route discovery overhead also increases with the increasing size of RZ. Thus, there exists a trade-off between latency of route determination and the message overhead.

Now, let us move to discuss the LAR-1 scheme. It uses an RZ that is rectangular in shape (Figure 2). It is assumed that $\mathrm{S}$ knows that $\mathrm{D}$ was at location $\left(X_{d}, Y_{d}\right)$ at time $t_{0}$. At time $t_{l}, \mathrm{~S}$ initiates a new route discovery for D. It is also assumed that $\mathrm{S}$ knows the speed $u$ with which $\mathrm{D}$ can move. Using this, $\mathrm{S}$ defines $\mathrm{EZ}$ at time $t_{1}$ to be the circle of radius $R_{e}=u\left(t_{1}-t_{0}\right)$ centred at location $\left(X_{d}, Y_{d}\right)$. As stated before, instead of the average speed, $u$ may be chosen to be the maximum speed or some other function of the speed distribution.

$\mathrm{RZ}$ is defined to be the smallest rectangle that includes current location of $\mathrm{S}$ and EZ, such that the sides of the rectangle are parallel to the $\mathrm{X}$ and $\mathrm{Y}$ axes. In Figure 2a, RZ is the rectangle whose corners are S, A, B and C, whereas in Figure 2b, the rectangle has corners at point A, B, $\mathrm{C}$ and $\mathrm{G}$. In Figure 2, current location of node $\mathrm{S}$ is denoted as $\left(X_{s}, Y_{s}\right)$, and consequently $\mathrm{S}$ can determine the four corners of RZ.

$S$ includes their coordinates with the RREQ packet transmitted when initiating route discovery. When a node receives a RREQ, it discards the request if the node is not within the rectangle specified by the four corners included in the RREQ. For instance, in Figure 2a, if node $i$ receives the RREQ from another node, node $i$ forwards the request to its neighbours, because $i$ determines that it is within the rectangular RZ. However, when node $j$ receives the RREQ, node $j$ discards the request, as node $j$ is not within RZ. 


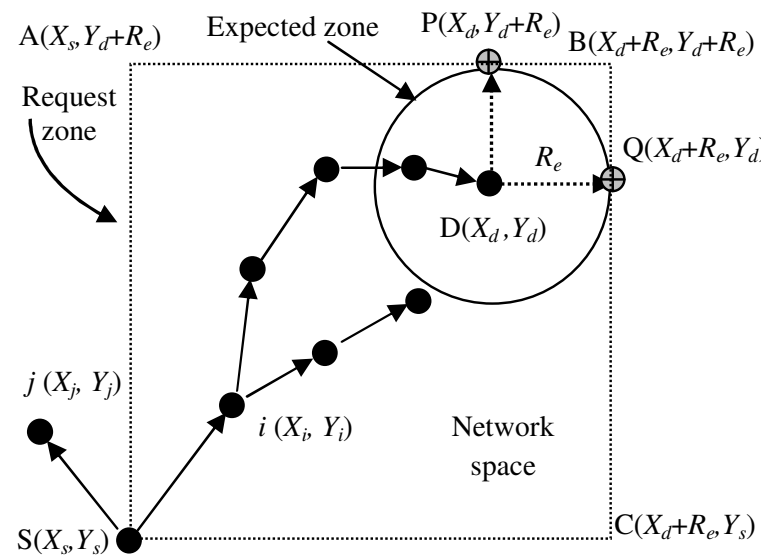

(a) Source node (S) outside the expected zone (EZ)

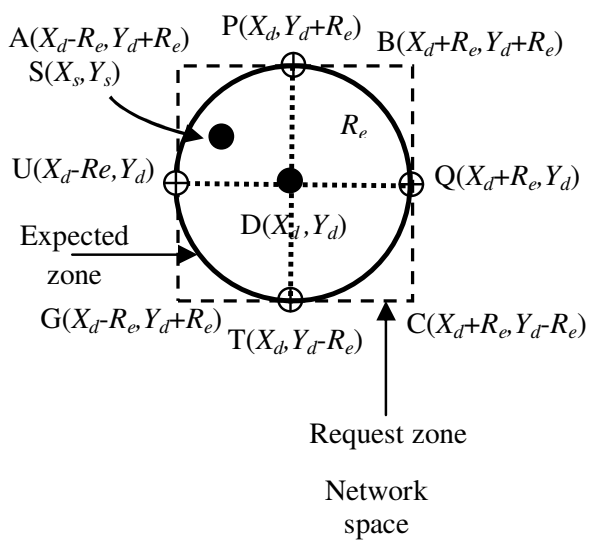

(b) Source node (S) within the expected zone (EZ).

Figure 2. The LAR-1 scheme.

When D receives the RREQ packet, it replies by sending a RREP packet (as in the pure flooding algorithm). However, in case of LAR-1, D includes its current location and current time in the RREP packet. When $S$ receives this RREP packet (ending its route discovery), it records the location of D. S can use this information to determine RZ for a future route discovery. It is also possible for $\mathrm{D}$ to include its current speed, or average speed over a recent time interval, with the RREP packet. This information could be used in a future route discovery. Further details on LAR schemes can be found in [8].

\subsection{Probabilistic Broadcast Algorithm}

Probabilistic algorithm is widely-used for flooding optimization during route discovery in MANETs. It aims at reducing number of retransmissions, in an attempt to alleviate the effects of the BSP in MANETs [5, 6, 11]. In this scheme, when receiving a RREQ packet, a node retransmits the packet with a certain $p_{t}$ and with probability $\left(1-p_{t}\right)$ it discards the packet. A node is allowed to retransmit a given RREQ packet only once, i.e., if a node receives a packet, it checks to see if it has retransmitted it before, if so then it just discards it, otherwise it performs its probabilistic retransmission check. Nodes usually identify the RREQ packet through its sequence number. The source node $p_{t}$ is always set to 1 , to enable the source initializing a new RREQ. While, $p_{t}$ for intermediate nodes (all nodes except the source and the destination) is determined using a static or dynamic approach. In static approach, a pre-determined $p_{t}$ (e.g., $p_{t}$ $\left.=P_{t}\right)\left(0 \leq P_{t} \leq 1\right)$ is set for each node on the networks. While, in dynamic approach, each node on the network locally calculates $p_{t}$ using certain probability distribution function of one or more independent variables. Figure 3 outlines the probabilistic broadcast algorithm.

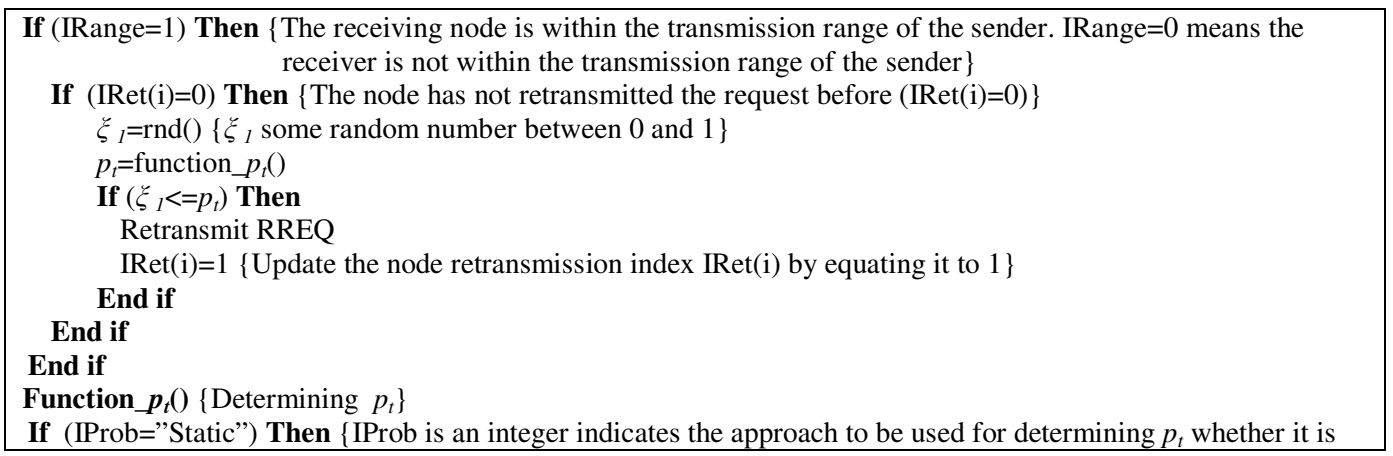


International Journal of Computer Networks \& Communications (IJCNC) Vol.3, No.6, November 2011

$\begin{aligned} & p_{t}=\text { constant value } \\ & \text { static or dynamic }\} \\ & \text { Else (IProb="Dynamic") } \\ & p_{t}=f(k)\end{aligned}$
End If

Figure 3. The probabilistic broadcast algorithm.

In dynamic probabilistic algorithm, $p_{t}$ is usually calculated as a function of the number of firsthop neighbors $(k)$, and it is referred to as $p_{t}(k)$. Many functions have been developed for calculating $p_{t}(k)[5,6,10,11,19,20,21,24]$. In this work, $p_{t}(k)$ function presented in AlBahadili [11] is considered. This is because it demonstrated an excellent performance in comparison with other distribution functions in various network conditions, which is expressed as [11]:

$$
p_{t}= \begin{cases}p_{\max } & \text { for } k \leq N_{1} \\ p_{1}-\frac{k-N_{1}}{N_{2}-N_{1}}\left(p_{1}-p_{2}\right) & \text { for } N_{1}<k<N_{2} \\ p_{\min } & \text { for } k \geq N_{2}\end{cases}
$$

Where $p_{t}(k)$ is the dynamic node retransmission probability; $k$ is the number of first-hop neighbor for the transmitting node; $p_{\min }$ and $p_{\max }$ are the minimum and maximum retransmission probabilities that could be assigned for a node; $N_{l}$ is the number of nodes at or below which $p_{t}$ is equal to $p_{\max } ; N_{2}$ is the number of nodes at or above which $p_{t}$ is equal to $p_{\min }, p_{1}$ and $p_{2}$ are the retransmission probabilities assigned to intermediate nodes surrounded by $k=N_{1}+1$ and $k=N_{2}-1$ nodes, respectively, $p_{1}$ and $p_{2}$ should lie between $p_{\max }$ and $p_{\min }$ (i.e., $p_{\max } \geq p_{1} \& p_{2} \geq p_{\min }$ ), and also $p_{1} \geq p_{2}$. Figure 4 shows the variation of $p_{t}$ with $k$. In general, selection of satisfactory distribution in the interval $\left[N_{1}+1, N_{2}-1\right]$ and the values of $p_{\max }, p_{\min }, p_{1}, p_{2}, N_{1}$, and $N_{2}$ depend on a number of factors and need to be carefully considered for every network condition. Details and evaluation of the above function are presented in [11].

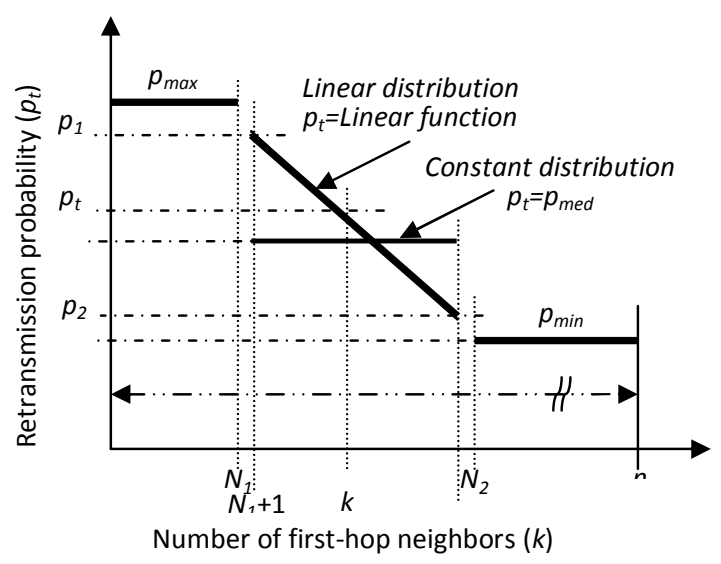

Figure 4. Retransmission probability as a function of $k$ [15].

\subsection{The LAR-1P Algorithm}

The description of LAR-1P algorithm is straightforward. It is simply, when receiving a broadcast message, a node within the RZ rebroadcasts the message with a pre-defined or dynamically calculated $p_{t}$, and each node is allowed to rebroadcast the received message only 
once. This contributes to a certain reduction in the number of retransmissions and consequently node average duplicate reception, thereafter the number of collisions and contentions at the receiver. The reduction in the number of retransmission will of course depend on the assigned $p_{t}$, and the algorithm is reduced to LAR-1 if the nodes' $p_{t}$ is set as unity.

It is important to recognize that reduction in the number of retransmission may introduce some reduction in network reachability, because if the number of intermediate nodes within RZ is small (low-density zones) and some of them will not rebroadcast the request message, which may result in a failure of delivery of the RREQ to destination, so that the source has to reinitiate a new RREQ. However, it is expected that if the number of intermediate nodes within RZ is large (high-density zones), there will be either only little (insignificant) or no reduction in network reachability. Figure 5 outlines the procedure for the LAR-1P algorithm.

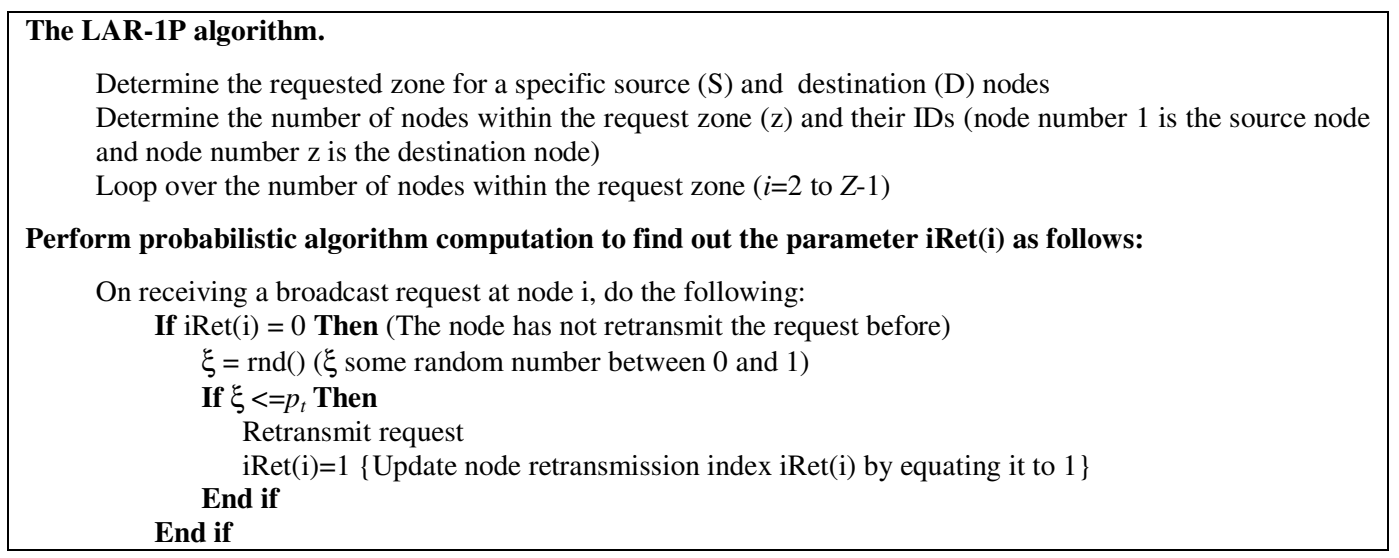

Figure 5. The LAR-1P algorithm.

In dynamic probability LAR-1P, there are two $k$ values that can be considered for calculating $p_{t}$ in Eqn. 1. The first value represents the total number of first-hop neighbors regardless of whether they are inside or outside RZ (same as in standard dynamic probabilistic algorithm); while the second value considers only those nodes inside RZ, which is usually equal to or less than the first value. Normally, every node on the network updates the lists (records) of its firsthop neighbors at the start of each time interval, which is usually lasted for what is referred to as the pause time $(\tau)$.

Now, considering the second value for calculating $p_{t}$ imposes extensive computation that needs to be performed by the node as each node needs to calculate $k\left(p_{t}\right)$ for every source-destination (S-D) pair during the same time interval; while for the first value assignment, a node needs to calculate $p_{t}$ only once for each time interval regardless of the S-D combinations.

It can be seen from the above description of LAR-1P that at high-density zones, the nodes will be given low $p_{t}$ s inflicting a reduction in the number of retransmissions that depends on the assigned $p_{t} \mathrm{~s}$. Which means the probabilistic algorithm is effective. While at low-density zones, the nodes will be allowed to retransmit the RREQ packet with high $p_{t}$, if it is unity or close to unity, then LAR-1P acts as LAR-1.

\subsection{Computation Procedures}

There are two computational procedures have been implemented in MANSim [13]. The first computational procedure computes the average parameters for a pre-specified S-D pair. In this procedure, the source node initiates $Q$ RREQs each time interval. The computation is repeated for a number of time intervals $(M)$, for each time interval, the nodes on the network are allowed 
to change their positions on the network. Therefore, the computed parameters should be averaged over $Q$ and $M$. The computed average values represent the average parameters associated with this particular S-D pair, but they may not reflect the average behavior of other $S-D$ pairs on the network. However, it has been found that for a network that has no probabilistic behavior, i.e., $p_{t}=1, Q$ has no effect on the computed parameters and $Q$ can be set to 1 .

The second computational procedure computes the average network parameters that can be considered as the average parameters for any S-D pair. This is calculated by looping over all nodes on the network as source nodes, and each source node tries to establish a route to all other nodes on the network as destination nodes. Furthermore, for each S-D pair, the source initiates $Q$ RREQs as described in the first computation procedure. The computed parameters for each source are averaged over $Q(N-1)$, and then the computed averaged values are averaged over $(N)$. In other words, the computed parameters are averaged over $(Q N(N-1))$.

In order to consider nodes mobility, the above calculations are repeated, in an outer loop of size $M$, and the results obtained for the computed parameters are averaged over $M$. In this case, the computed parameters may well represent the average behavior of any of the nodes on the network. For non-mobile nodes (fixed nodes), $M$ has no effect on the computed parameters and can be set to 1. Figure 6 outlines the second computation procedure.

Loop over the number of time intervals $(M)(t=1, M)$

Loop over the number of nodes $(N)$ as source nodes $(s=1, N)$

Loop over the number of nodes $(N)$ as destination nodes $(d=1, n)$, except for $s=d$

Loop over the number of transmitted request message $(q=1, Q)$

Compute IRec() and IRet()

Compute the average values of the computed parameters (over $Q$ ) for $s$ - $d$ pair

Compute the average values of the computed parameters (over $N$-1) for node $s$

Compute the average values of the network parameters (over $N$ ) for time interval $t$

Compute the average values of the computed network parameters (over $M$ ).

Figure 6. Second computational procedure in MANSim.

\section{RESUltS AND Discussions}

The LAR-1P algorithm is implemented on the MANET simulator (MANSim), which is especially developed network simulator for evaluating the performance of various route discovery algorithms in MANETs [13]. It consists of four major modules: network (geometrical), mobility, computational, and algorithm modules. Detail description of these modules of MANSim is given in [13]. The performance of the algorithms is evaluated in terms of two parameters, namely, the number of retransmissions (RET) and reachability (RCH). RET is defined as the average number of retransmissions normalized to the total number of nodes on the network $(N)$. RCH is defined as the average number of reachable nodes by any node on the network normalized to $N$; or the probability by which a RREQ packet will be successfully delivered from source to destination [7].

In order to investigate the effect of nodes densities (number of nodes divided by the network area, i.e., $N_{d}=N / A$ ) on the performance of the LAR-1P algorithm, a number of simulations were performed using MANSim. The network environment and simulation setup can be described as follows: Each simulation starts by generating $N$ nodes randomly distributed across a network area $(A)$ of $500 \times 500 \mathrm{~m}$ using a uniform probability distribution function described in [13]. In this work the number of nodes considered is: 50, 75, 100, 125, and 150 nodes. All nodes are assumed to be transmitting with the same radio transmission range $(R)$ of $100 \mathrm{~m}$ and move with 
an average velocity $(u)$ of $5 \mathrm{~m} / \mathrm{sec}$. The mobility model used is the random waypoint mobility model $[29,30]$.

One important parameter that needs to be carefully specified to obtain an adequate network performance is the pause time $(\tau)$, which is defined as a period of time in which all nodes on network are assumed motionless but continue transmitting [31]. In general, it has been found that $\tau$ must be chosen so that the distance traveled by a node after any location update interval is less than $R$. In MANSim, it is calculated as: $\tau=0.75^{*} R / u$, so that for $R=100 \mathrm{~m}$ and $u=5 \mathrm{~m} / \mathrm{sec}$, $\tau$ is equal to $15 \mathrm{sec}$. The simulation time $\left(T_{\text {sim }}\right)$ is divided into a number of intervals (or we call mobility loops $(M)$ ) of period $\tau$, thus the relationship between $T_{\text {sim }}, \tau$, and $M$ is expressed as $T_{s i m}=\tau M$. In all simulations, $T_{\text {sim }}$ is $1800 \mathrm{sec}$ giving 120 mobility loops. The input data for this scenario is summarized in Table 1 .

\begin{tabular}{|l|l|}
\hline \multicolumn{2}{|c|}{$\begin{array}{c}\text { Table } 1 . \\
\text { Input data. }\end{array}$} \\
\hline \multicolumn{1}{|c|}{ Parameters } & \multicolumn{1}{c|}{ Value } \\
\hline Geometrical model & Random node distribution \\
\hline Network area & $500 \times 500 \mathrm{~m}$ \\
\hline Number of nodes $(n)$ & $50,75,100,125,150$ nodes \\
\hline Transmission radius $(R)$ & $100 \mathrm{~m}$ \\
\hline Average node speed $(u)$ & $5 \mathrm{~m} / \mathrm{sec}$ \\
\hline Simulation time $\left(T_{\text {sim }}\right)$ & 1800 \\
\hline Pause time $(\tau)$ & $15 \mathrm{sec}$ \\
\hline Mobility loop size $(n M o b s)$ & 120 \\
\hline
\end{tabular}

Furthermore, in order to compare the performance of LAR-1P with other route discovery algorithms, we use MANSim to estimate the performance (RET and $\mathrm{RCH}$ ) of pure flooding, dynamic probabilistic, and LAR-1 (LAR-1P with $\left.p_{t}=1\right)$ using the same network environment and simulation setup. The results obtained are listed in Table 2.

\begin{tabular}{|c|c|c|c|c|c|c|}
\hline \multicolumn{7}{|c|}{$\begin{array}{rr}\text { Table } 2 \\
\text { Comparing the nerformance of LAR- }\end{array}$} \\
\hline$N$ & $\begin{array}{c}N_{d} \\
(N / A)\end{array}$ & $\begin{array}{c}\text { Pure } \\
\text { Flooding }\end{array}$ & $\begin{array}{c}\text { Dynamic } \\
\text { Probabilistic }\end{array}$ & LAR-1 & LAR-1P & $\begin{array}{c}\mathrm{R}_{\mathrm{RET}}-\mathrm{R}_{\mathrm{RCH}} \\
(\%)\end{array}$ \\
\hline \multicolumn{7}{|c|}{ RET } \\
\hline 50 & $2 \times 10^{-4}$ & 0.715 & 0.652 & 0.087 & 0.084 & 3.45 \\
\hline 75 & $3 \times 10^{-4}$ & 0.932 & 0.771 & 0.129 & 0.108 & 16.28 \\
\hline 100 & $4 \times 10^{-4}$ & 0.975 & 0.718 & 0.147 & 0.115 & 21.77 \\
\hline 125 & $5 \times 10^{-4}$ & 0.983 & 0.643 & 0.159 & 0.115 & 27.67 \\
\hline 150 & $6 \times 10^{-4}$ & 0.987 & 0.589 & 0.161 & 0.109 & 32.30 \\
\hline \multicolumn{7}{|c|}{ RCH } \\
\hline 50 & $2 \times 10^{-4}$ & 0.767 & 0.750 & 0.396 & 0.394 & 0.51 \\
\hline 75 & $3 \times 10^{-4}$ & 0.961 & 0.946 & 0.641 & 0.612 & 4.52 \\
\hline 100 & $4 \times 10^{-4}$ & 0.995 & 0.981 & 0.772 & 0.720 & 6.74 \\
\hline 125 & $5 \times 10^{-4}$ & 0.999 & 0.990 & 0.857 & 0.813 & 5.13 \\
\hline 150 & $6 \times 10^{-4}$ & 1.000 & 0.995 & 0.894 & 0.859 & 3.91 \\
\hline
\end{tabular}

Before, we proceed with the discussion of the results in Table 2, let us remind ourselves with three important points, the first one is that the main requirement of any route discovery algorithm is to achieve the highest possible network reachability at minimum number of retransmissions, or reduces the number of retransmissions without sacrificing the network reachability. The second point is that the results in Table 2 present the average network behavior over $1800 \mathrm{sec}$ simulation time. Finally, $p_{t}$ in LAR-1P is calculated considering the first-hop neighbors lies inside the request zone only. 
For each nodes density, the percentage reduction in average network reachability is calculated by: $\left(\mathrm{R}_{\mathrm{RCH}}=100 \times\left(\mathrm{RCH}_{\mathrm{LAR}-1}-\mathrm{RCH}_{\mathrm{LAR}-1 \mathrm{P}}\right) / \mathrm{RCH}_{\mathrm{LAR}-1}\right)$. Similarly, the percentage reduction in number of retransmissions is calculated as: $\left(\mathrm{R}_{\mathrm{RET}}=100 \times\left(\mathrm{RET}_{\mathrm{LAR}-1}-\mathrm{RET}_{\mathrm{LAR}-1 \mathrm{P}}\right) / \mathrm{RET}_{\mathrm{LAR}-1}\right)$. It can be seen from Table 2 that the performance of LAR-1P varies in the same pattern as other algorithms, where the reachability significantly improves with increasing nodes densities at relatively slight increase in the number of transmissions. Furthermore, it can be easily deduced that in comparison with LAR-1, $\mathrm{R}_{\mathrm{RCH}}$ fluctuated around a very small percent with increasing nodes densities, while $\mathrm{R}_{\mathrm{RET}}$ increasing, which means more reduction in number of collisions, contentions, and power consumption.

Table 3 presents the average $p_{t}\left(p_{t, \text { avg }}\right)$ and the average number of first-hop neighbors $\left(k_{\text {avg }}\right)$ for the dynamic probabilistic and LAR-1P algorithms. It shows that, for both dynamic probabilistic and LAR-1P, $k_{\text {avg }}$ is increasing with increasing nodes densities, and for each node density, $k_{a v g}$ for dynamic probabilistic is greater than that for LAR-1P. This is because in LAR-1P we only consider first-hop neighbors that are positioned inside the request zone. As a result of that and in accordance with Eqn. 1, $p_{t, a v g}$ is decreasing with increasing nodes densities for the two algorithms, and for the same node density, $p_{t, a v g}$ for dynamic probabilistic is less than that for LAR-1P.

\begin{tabular}{|c|c|c|c|c|c|}
\hline \multicolumn{6}{|c|}{ Table 3} \\
Comparing $\boldsymbol{p}_{t, \text { avg }}$ and $\boldsymbol{k}_{\text {avg }}$ for dynamic probabilistic and LAR-1P for various nodes densities. \\
\hline \multirow{2}{*}{$N$} & \multirow{2}{*}{$\begin{array}{c}N_{d} \\
(N / A)\end{array}$} & $\begin{array}{c}\text { Dynamic } \\
\text { Probabilistic }\end{array}$ & LAR-1P & $\begin{array}{c}\text { Dynamic } \\
\text { Probabilistic }\end{array}$ & LAR-1P \\
\cline { 3 - 6 } & & 0.935 & 0.981 & 4.913 & 3.418 \\
\hline 50 & $2 \times 10^{-4}$ & 0.844 & 0.946 & 7.262 & 4.945 \\
\hline 75 & $3 \times 10^{-4}$ & 0.749 & 0.904 & 9.562 & 6.247 \\
\hline 100 & $4 \times 10^{-4}$ & 0.661 & 0.848 & 12.092 & 7.865 \\
\hline 125 & $5 \times 10^{-4}$ & 0.601 & 0.796 & 14.373 & 9.401 \\
\hline 150 & $6 \times 10^{-4}$ & \multicolumn{2}{|c|}{} \\
\hline
\end{tabular}

\subsection{Analyzing the Performance of the LAR-1P Algorithm}

In what follows we shall analyze the performance of the algorithms for a single node density value of $4 \times 10^{-4}$ nodes $/ \mathrm{m}^{2}$. In this case, pure flooding provides the highest network reachability of $99.5 \%$ (almost $100 \%$ ) at $97.5 \%$ of the nodes on the network (almost all intermediate nodes) engaged in RREQ packets retransmission during the route discovery phase resulting in significant collisions and contention. The dynamic probabilistic algorithm achieves a very high reachability of $98.1 \%$ but only reduces the number of retransmissions by $\sim 28 \%$ to around $72 \%$.

The LAR-1 algorithm reduces the average number of retransmission to a very low level of $14.7 \%$ at the cost of reducing average network reachability to $77.2 \%$. In other words, LAR-1 introduces a significant reduction of $\sim 85 \%$ in the average number of retransmissions but at the same time reducing network reachability by $\sim 22 \%$. However, these results represent an average behavior over uniform distribution of zones densities. But if most of route discovery processes involve searching the route in high-density zones, then the average number of retransmissions will be definitely higher than $14.7 \%$. For example, for request zones that confine all nodes, the network reachability and number of retransmissions will be the same as or close to that in pure flooding. Furthermore, in high-density zones the delay is much higher than in low-density zones.

Now, let us move to discuss the results for LAR-1P. As it can be seen in Table 2 that LAR-1P provides, on network scale, a network reachability of 5.2\% less than LAR-1 and reduces the associated number of redundant retransmissions by $3.2 \%$. This is, in fact, not a very impressive achievement to consider LAR-1P as a new competitor flooding optimization algorithm. 
However, when we look at zone scale, for example, for high-density zones, LAR-1P attains the performance of dynamic probabilistic of $98.1 \%$ reachability at $\sim 30 \%$ reduction in the number of retransmissions. Thus, if most route discovery processes involve high-density zones, a reduction in the number of retransmissions is always equal to or close to $30 \%$ yielding higher average reduction in the number of retransmissions. On the other hand, for low-density zones, the estimated retransmission probabilities are always close to unity, i.e., inflicting pure flooding within the request zone, which means LAR-1 is the acting algorithm.

In order to closely demonstrate the advantage of LAR-1P, let us consider the following example. Assume node 1 is the source node and all other nodes are examined as destination nodes one at a time. For each S-D pair we count the number of nodes within the request zone $\left(N_{z}\right)$ including the source and the destination nodes. Then, we count the number of cases in which $N_{z}$ lies within the ranges $\left.2-10,11-20,21-30, \ldots, 91-100\right)$. The minimum is 2 , which means only the source and the destination nodes are present within the request zone and no existence for intermediate nodes. The maximum is 100 (total number of nodes on the network), which means all nodes are confined into the request zone. For each range we estimate the reachability and number of retransmissions for both LAR-1 and LAR-1P algorithms. Also, we estimate $p_{t, a v g}$ and $k_{\text {avg. }}$. The results obtained are summarized in Table 4 .

\begin{tabular}{|c|c|c|c|c|c|c|c|c|c|c|c|}
\hline \multicolumn{12}{|c|}{$\begin{array}{l}\text { Table } 4 . \\
\text { Comparison between the LAR-1 and LAR-1P }\end{array}$} \\
\hline \multirow{2}{*}{$N_{z}$} & \multirow{2}{*}{$Z$} & \multicolumn{5}{|c|}{ LAR-1 } & \multicolumn{5}{|c|}{ LAR-1P } \\
\hline & & RET & $\mathrm{RCH}$ & $\mathrm{RCH}$ & $p_{t, a v g}$ & $k_{\text {avg }}$ & RET & $\mathrm{RCH}$ & $\mathrm{RCH}$ & $p_{t, \text { avg }}$ & $k_{\text {avg }}$ \\
\hline $2-10$ & 40 & 0.048 & 32 & 0.800 & 1.000 & 3.873 & 0.043 & 31 & 0.775 & 0.967 & 3.873 \\
\hline $11-20$ & 27 & 0.127 & 22 & 0.815 & 1.000 & 5.564 & 0.113 & 21 & 0.778 & 0.914 & 5.564 \\
\hline $21-30$ & 11 & 0.222 & 10 & 0.909 & 1.000 & 6.832 & 0.186 & 10 & 0.909 & 0.868 & 6.832 \\
\hline $31-40$ & 11 & 0.328 & 11 & 1.000 & 1.000 & 8.828 & 0.263 & 11 & 1.000 & 0.787 & 8.828 \\
\hline $41-50$ & 10 & 0.431 & 10 & 1.000 & 1.000 & 9.515 & 0.310 & 10 & 1.000 & 0.756 & 9.515 \\
\hline
\end{tabular}

Now, let us explain the results in Table $4 . \mathrm{Z}$ in the second column gives the number of request zones that contains a number of nodes within the range indicated in the first column. For example, the number of request zones that contains a number of nodes within the range from 2 to 10 is 40 request zones, and 27 request zones contains a number of nodes between 11 to 20 nodes.

For LAR-1, consider the case of low-density zone (2 to 10 nodes), in 32 out of the 40 request zones, the source and the destination were able to establish a connection and 8 of them were not, giving a reachability of $80 \%$. Due to the small number of nodes within the request zones, the number of retransmissions was only $4.8 \%$. The LAR-1P provides slightly less reachability and less number of retransmissions. For high-density zone (41 to 50 nodes), the LAR-1P accomplishes the same reachability 10 out of 10 at a significant reduction in the number of retransmissions from $43.1 \%$ for the LAR- 1 to $31 \%$, and that what LAR-1P is all about. In highzones densities, reduces the number of retransmissions significantly and maintains the same network reachability. 


\section{Conclusions}

This paper provides a detail description of the main concept of a new route discovery algorithm in MANET, namely, the LAR-1P algorithm, which combines two well-known route discovery algorithms: The location-aided routing scheme 1 (LAR-1) and dynamic probabilistic algorithms. The simulation results demonstrated that the performance of the LAR-1P over the LAR-1 is improving with increasing nodes densities, i.e., more reduction in the number of retransmissions can be achieved against insignificant reduction in network reachability. Moreover, the performance of the LAR-1P algorithm overwhelmed the performance of LAR-1 in high-zones densities, while almost provides the performance of LAR-1 in low-zones densities.

As recommendations for future work, we recommend to investigate the effects of nodes speeds on the performance of the LAR-1P algorithm. In addition, it will be very useful to perform performance evaluation of the algorithm using different distribution functions for calculating intermediate nodes retransmission probabilities. Furthermore, we recommend evaluating the performance of the new algorithm in terms of load, through, and delay using well-known network simulators, such OPNET, NS2, NS3, or GloMoSim.

\section{REFERENCES}

[1] Hussein Al-Bahadili. A New Route Discovery Algorithm in MANETs Combining Location-Aided Routing and Probabilistic Algorithms. Submitted to The Mediterranean Journal of Computers and Networks (MEDJCN) on $26^{\text {th }}$ August 2011.

[2] D. B. Johnson and D. A. Maltz. Dynamic Source Routing in Ad Hoc Wireless Networks. In Mobile Computing (Editors: T. Imielinski and H. Korth, Publisher: Kluwer Academic Publishers), pp. 153-181, 1996.

[3] C. Perkins and E. Royer. Ad Hoc on Demand Distance Vector Routing. Proceedings of the $2^{\text {nd }}$ IEEE Workshop on Mobile Computing Systems and Applications (WMCSA'99), pp. 90-100, New Orleans, LA, USA, 1999.

[4] Z. J. Haas, M. R. Pearlman, and P. Samar. The Zone Routing Protocol (ZRP) for Ad Hoc Networks. Mobile Ad Hoc Network Working Group, IETF Internet Draft, 2002. Retrieved on 10 October 2009 from http://tools.ietf.org/id/draft-ietf-manet-zone-zrp-04.txt.

[5] M. Bani-Yassein, and Ould-Khaoua. Applications of Probabilistic Flooding in MANETs. International Journal of Ubiquitous Computing and Communication, Vol. 1, Issue 1, pp. 1-5, 2007.

[6] M. Bani-Yassein, M. Ould-Khaoua, L. M. Mackenzie, and S. Papanastasiou. Performance Analysis of Adjusted Probabilistic Broadcasting in Mobile Ad Hoc Networks. International. Journal of Wireless Information Networks, Vol. 13, No. 2, pp. 127-140, 2006.

[7] S. Tseng, S. Ni, Y. Chen, and J. Sheu. The Broadcast Storm Problem in a Mobile Ad Hoc Network. Journal of Wireless Networks, Vol. 8, No. 2, pp. 153-167, 2002.

[8] Y. B. Ko and N. H. Vaidya. Location-Aided Routing (LAR) in Mobile Ad Hoc Networks. Wireless Networks, Vol. 6, No. 4, pp. 307-321, 2000.

[9] J. Boleng and T. Camp. Adaptive Location-Aided Mobile Ad Hoc Network Routing. Proceedings of the $23^{\text {rd }}$ IEEE International Performance, Computing, and Communications Conference (IPCCC), pp. 423-432, Phoenix, Arizona, USA, 2004.

[10] Z. J. Haas, J. Y. Halpern, and L. Li. Gossip-Based Ad Hoc Routing. IEEE/ACM Transactions on Networking, Vol. 14, Issue 3, pp. 479-491, 2006.

[11] Hussein Al-Bahadili. Enhancing the Performance of Adjusted Probabilistic Broadcast in MANETs. The Mediterranean Journal of Computers and Networks (MEDJCN), Vol. 6, No. 4, pp. 138-144, 2010. 
International Journal of Computer Networks \& Communications (IJCNC) Vol.3, No.6, November 2011

[12] Hussein Al-Bahadili and Khaled Kaabneh. Analyzing the Performance of Probabilistic Algorithm in Noisy MANETs. International Journal of Wireless \& Mobile Networks (IJWMN), Vol. 2, No. 3, pp. 83-95, August 2010.

[13] Hussein Al-Bahadili. On the Use of Discrete-Event Simulation in Computer Networks Analysis and Design. In Handbook of Research on Discrete-Event Simulation Environments: Technologies and Applications (Editors: Evon Abu-Taieh and Asim El-Sheikh), Chapter 19, pp. 414-442, Information Science Reference, PA, USA, 2010.

[14] Hussein Al-Bahadili, Omar Al-Basheer, and Amjad Al-Thaher. A Location Aided RoutingProbabilistic Algorithm for Flooding Optimization in MANETs. Proceedings of Mosharaka International-Conference on Communications, Networking and Information Technology (MICCNIT 2007), Amman-Jordan, 6-8 December 2007.

[15] Amjad F. Al-Thaher. A Location-Aided Routing-Probabilistic Protocol for Flooding Optimization in Mobile Ad Hoc Networks. M.Sc Thesis. Amman Arab University for Graduate Studies, Graduate College of Computing Studies, Amman-Jordan, June 2007.

[16] J. Li, J. Jannotti, D. Couto, D. Karger and R. Morris. A Scalable Location Service for Geographic Ad Hoc Routing. Proceedings of the $6^{\text {th }}$ International Conference on Mobile Computing and Networking (MOBICOM 00), pp. 120-130, United State, 2000.

[17] K. Zeng, K. Ren, and W. Luo. Geographic On-Demand Disjoint Multipath Routing in Wireless Ad hoc Networks. Proceedings of IEEE Military Communication Conference (MILCOM 05), United State, 2005.

[18] N. Vyas, and I. Mahgoub. Location and Mobility Pattern Based Routing Algorithm for Mobile Ad Hoc Wireless Networks. Proceedings of the 2003 International Symposium on Performance Evaluation of Computer and Telecommunication Systems (SPECTS 03), 2003.

[19] Y. Sasson, D. Cavin, and A. Schiper. Probabilistic Broadcast for Flooding in Wireless Mobile Ad Hoc Networks. In Proceedings of IEEE Wireless Communications and Networking (WCNC'03): Vol. 2, pp. 1124-1130. New Orleans, LA, USA.

[20] J. S. Kim, Q. Zhang, and D. P. Agrawal. Probabilistic Broadcasting Based on Coverage Area and Neighbor Confirmation in Mobile Ad Hoc Networks. In Proceedings of the IEEE Global Telecommunications Conference Workshops (GlobeCom'04), pp. 96-101, Dallas, Texas, USA. The IEEE Society Press, 2004.

[21] D. Scott and A. Yasinsac. Dynamic Probabilistic Retransmission in Ad Hoc Networks. In H. R. Arabnia, L. T. Yang, and C. H. Yeh (Eds.), Proceeding of the International Conference on Wireless Networks (ICWN'04): Vol. 1, pp. 158-164. Las Vegas, Nevada, USA, CSREA Press, 2004.

[22] C. Barrett, S. Eidenbenz, L. Kroc, M. Marathe, and J. Smith. Parametric Probabilistic Routing in Sensor Networks. Journal of Mobile Networks and Applications, Vol. 10, Issue 4, pp. 529-544, 2005.

[23] K. Viswanath, and K. Obraczka. Modeling the Performance of Flooding in Wireless Multi-Hop Ad Hoc Networks. Journal of Computer Communications, Vol. 29, No. 8, pp. 949-956, 2005.

[24] Q. Zhang and D. P. Agrawal. Dynamic Probabilistic Broadcasting in MANETs. Journal of Parallel and Distributed Computing, Vol. 65, No. 2, pp. 220-233, 2005.

[25] J. Abdulai, M. Ould-Khaoua, L. Mackenzie, and M. Bani-Yassin. On the Forwarding Probability for On-Demand Probabilistic Route Discovery in MANETs. Proceedings of the $22^{\text {nd }}$ Annual UK Performance Engineering Workshop (UKPEW'06), Poole, Dorset, UK, pp. 9-15, 2006.

[26] J. Abdulai, M. Ould-Khaoua, and L. Mackenzie. Improving Probabilistic Route Discovery in Mobile Ad Hoc Networks. Proceedings of the $32^{\text {nd }}$ IEEE Conference on Local Computer Networks (LCN '07), Dublin, Ireland, IEEE Computer Society, pp. 739-746, 2007. 
International Journal of Computer Networks \& Communications (IJCNC) Vol.3, No.6, November 2011

[27] I. Khan, A. Javaid, H. Qian. Coverage-Based Dynamically Adjusted Probabilistic Forwarding for Wireless Mobile Ad Hoc Networks. Proceedings of the $1^{\text {st }}$ ACM International Workshop on Heterogeneous Sensor and Actor Networks (HeterSanet '08), pp. 81-88, Hong Kong, China, 2008.

[28] A. Hanash, A. Siddique, I. Awan, M. Woodward. Performance Evaluation of Dynamic Probabilistic Broadcasting for Flooding in Mobile Ad Hoc Networks. Journal of Simulation Modeling Practice and Theory, Vol. 17, No. 2, pp. 364-375, 2009.

[29] Guolong Lin, Guevara Noubir, and Rajmohan Rajaraman. Mobility Models for Ad hoc Network Simulation. Proceedings of the $23^{\text {rd }}$ Annual Joint Conference of the IEEE Computer and Communications Societies (INFOCOM 2004). Hong Kong, China, March 7-11, 2004.

[30] T. Camp, J. Boleng, and V. Davies. A Survey of Mobility Models for Ad Hoc Network Research. Wireless Communications \& Mobile Computing (WCMC): Special Issue on Mobile Ad Hoc Networking: Research, Trends and Applications, Vol. 2, No. 5, pp. 483-502, 2002.

[31] Dhananjay Bisen, Preetam Suman, Sanjeev Sharma, and Rajesh Shukla. Effect of Pause Time on DSR, AODV and DYMO Routing Protocols in MANET. International Journal of IT \& Knowledge Management, Vol. 3, No. 1, Article 8, 2010.

\section{AUTHORS}

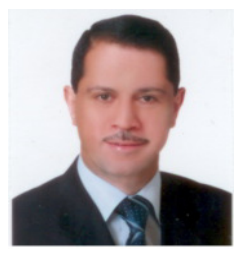

Hussein Al-Bahadili (Hbahadili@uop.edu.jo) is currently an associate professor at Petra University, Faculty of Information Technology, Amman-Jordan. He received his B.Sc degree in Engineering from College of engineering (University of Baghdad, Iraq) in 1986. He received his M.Sc and $\mathrm{PhD}$ degrees in Engineering from Queen Mary College (University of London, UK) in 1988 and 1991, respectively. His field of study was parallel computers. He is a visiting researcher at the Wireless Networks and Communications Centre (WNCC) at University of Brunel, UK. He is also a visiting researcher at the School of Engineering, University of Surrey, UK. He has published many papers and book chapters in different fields of science and engineering in numerous leading scholarly and practitioner journals, books, and presented at leading world-level scholarly conferences. He is an editor for a book titled: Simulation in Computer Network Design and Modeling: Use and Analysis published by IGI-Global. Till now he published four novel algorithms in data compression, route discovery in mobile ad hoc network, trust determination in wireless networks, and, finally, a novel compressed index-query Web search engine model. His research interests include parallel and distributed computing, wireless communications, computer networks, cryptography and network security, data compression, image processing, and artificial intelligence and expert systems.

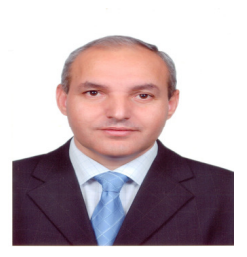

Ali Maqousi (amaqousi@uop.edu.jo). He is an assistance professor at Petra University, Faculty of Information Technology, Amman-Jordan. He is acting as a head of the department of Computer Science and Computer Networks. He received his $\mathrm{PhD}$ in computer science from Oxford Brookes University, UK, 2003 for his work on providing Quality of Service (QoS) in packet switched networks. He was a network administrator and part-time teacher assistant at Petra University (PU) from 1993-1997 and full-time teacher assistant from 1999-2003. Since 2003 he is an assistant professor at the Faculty of Information Technology at PU and currently he is the head of computer science and networking department. He is ITSAF Secretary - General (Information Technology Students Activity Fair, ITSAF is a yearly event for University students since 2005). He is the university liaison officer for European Union $7^{\text {th }}$ framework program (FP7) and Tempus since 2007. He is involved in research relating to multi-service networking, network performance, security and privacy, and social networks. 